\title{
The Hewlett-Packard Europhysics Prize
}

\author{
for Outstanding Achievement In Solid-State Physics
}

The establishment of the Hewlett-Packard Europhysics Prize (H-PEP) dates back to 1974 when the Hewlett-Packard company from Palo Alto in California, which had set up its first European manufacturing plant in 1959, was enjoying a period of rapid expansion. As a member of the Board of Hewlett-Packard (Europe) SA, the European subsidiary, I was present when the President of the Board, Richard Alberding, announced that the company wished to contribute to the life of Geneva, home of the European headquarters, by providing occasional help to some of the Canton's many international organizations. The EPS was a logical choice: the importance of European physics was unquestioned and the young society had proven to be vigorous and viable. It was also one of the few societies which joined together both eastern and western Europe.

There existed, of course, venerable international awards in physics, and various countries had national prizes, but a European prize was an innovation. The quality of the Selection Committee would be decisive and the presence of a solid sponsor was a key element. Initial reception of the Prize by the European physics community was not unanimous. All kinds of valid questions were asked, and many helpful suggestions offered. Our main answer was that the Prize made no claim to exclusivity: EPS welcomed other awards in other fields from other sources. Time has passed; several prizes have joined the Society, and the uniqueness of the first award has become clear to friends and sceptics alike.

The Prizes's Charter states that: "The Award shall be given in recognition of recent work by one or more individuals in the area of physics of condensed matter, specifically works leading to advances in the fields of electronic, electrical and materials engineering which, in the opinion of the Society's Selection Committee, represents scientific excellence. "Recent work" is defined to mean completed within five years prior to the Award. The Award may be given for either pure or applied research at the discretion of the Society."

The notion of "recent work" was to play a central rôle, while that of "pure research" have given rise to discussion both inside and outside the committee, as has the reference to "engineering advances". We feel that these notions are difficult but important for the development of physics. My own view is that fundamental research is judged by academic authorities, and applied research by people with responsibilities in manufacturing. So for the working physicist, it makes a large difference whether the goal is increased knowledge, or increased profit. But for a prize committee, this distinction is not decisive since what counts above all is scientific excellence.

At the beginning, there were two Nobel Prizes which had been recognized previously by the H-PE Prize (K. von Klitzing, 1982; G.K. Binnig and $\mathrm{H}$. Rohrer, 1984). Next came the discovery of high-temperature superconductivity, where everything went very quickly: nominations were made independently and the H-PE Prize announced first, although the Nobel Prize bears an earlier date. And then follow two Nobel Prizes in physics and one in chemistry that could have fallen under the objectives of the H-PE Prize. There is no harm that the choices of the prizes do not coincide. Nobel awards might still fall to one of our laureates; less likely is that the H-PE Prize will go

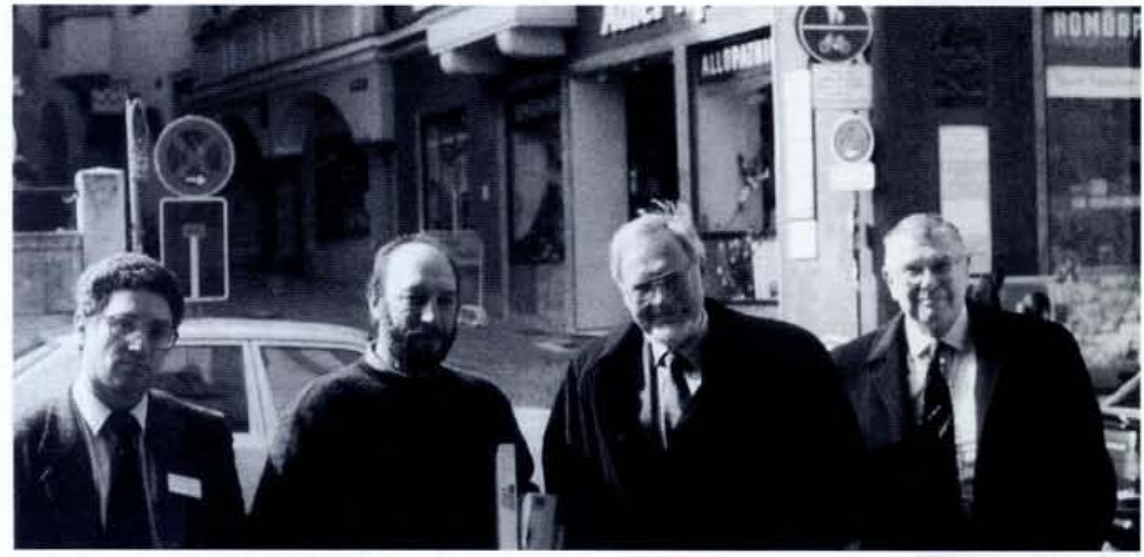

From left to right, D.E. Khmelnitskii and B.Z. Spivak (two of the five 1993 Hewlett-Packard Europhysics prizewinners), and A. Staufer and L. Cutler (from Hewlett-Packard) at the 1993 General Conference of the Condensed Matter Division of EPS in Regensburg.

to a Nobel Laureate as our award is out to discover new talent. Looking at the list of prizewinners, it is however difficult to avoid noticing a certain evolution.

Physics confers benefit to mankind by developing diagnostic tools, from X-ray and tracer diagnostics to different kinds of tomographs, from the electron microscope to tunnelling microscopy. There are also the various spectroscopies (neutron, meson, positron, etc.), photo-emission in all its varieties, and point-contact microscopy. Another major development is the emergence of numerical algorithms, often based on the local-density approximation, allowing ever more reliable numerical predictions of physical properties. And then there are new phases such as the condensation of excitons; and superconductors (organic, sulphuric, oxidic, carbonic), different forms of hydrogen, amorphous phases, and phases with heavy fermions and valence fluctuations. Finally, we have all the surprising, and useful, twodimensional configurations, such as heterojunctions, surface waves, and inversion layers supporting the anomalous Hall resistance with its enormous contributions to metrology. Thanks to modern optics with its optical molasses and fountains, frequency measurement, the basis of metrology, is moving to new horizons. Perhaps the time will come when optics should be added to the Award's Charter. Meanwhile, electro-optics and the applications of lasers need not be excluded.

Since the immediate post-war period when so much had to be rebuilt there has been continuous progress, sometimes rewarded by real breakthroughs, both in western and eastern Europe. It is one of the merits of EPS and the H-PE Prize that they have encouraged the necessary cooperation and recognition between the two parts of Europe during a time of mistrust.

The Prize is now a living part of our community. Geneva and EPS greatly appreciate that Hewlett-Packard provides a permanent and secure material basis. We should also thank representatives from Palo Alto who have never shied away from the long trip to Selection Committee meetings: B. Olivier, D. Hammond and Len Cutler, not forgetting Arnold Staufer who has guided the Prize within Hewlett-Packard (Europe).

M. Peter, University of Geneva
Condensation of excitons ('75):

V.S. Bagaev, L.V. Keldysh

J.E. Pokrovsky, M. Voos

Physics of liquid crystals ('76):

W. Helfrich

Amorphous silicon devices ('77):

W.E. Spear

Heterojunctions ('78):

Zh.I. Alferov

Surface acoustic wave devices

('79): E.A. Ash, J.H. Collins,

Y.V. Gulaev,

K.A. Ingebrigtsen,

E.G.S. Paige

Calculation of electronic properties of materials ('80):

O.K. Andersen,

A.R. Miedema

Quantized Hall resistance ('82):

K. von Klitzing

Atom and solid hydrogen ('83):

A.F. Silvera

Scanning tunnelling microscope

('84): G.K. Binnig,

H. Rohrer

Low-dimensional physics ('85):

J. Als-Nielsen, M. Pepper

Neutron spin echo spectroscopy

('86): F. Mezei

Point-contact spectroscopy ('87):

I.K. Yanson

High $-T_{c}$ superconductivity ('88):

J.G. Bednorz,

K.A. Müller

Heavy-fermion metals ('89):

F. Steglich, H.-R. Ott,

G.G. Lonzarich

ab initio calculation of molecu-

lar dynamics ('90):

R. Car, M. Parrinello

Organic metals ('91):

K. Beechgard, D. Jérome

Surface structures ('92):

G. Ertl, H. Ibach,

J.P. Toennies

Coherent phenomena in disordered conductors ('93):

B.L. Altshuler, A.G.

Aronov, A.I. Larkin, D.E.

Khmelnitskii, B.Z. Spivak 